\title{
Channel Estimation in OFDM System using Compressive Sensing Framework: A Review \\ ${ }^{* 1}$ Prachi Rane, ${ }^{2}$ Smita Daware \\ ${ }^{1,2}$ Department of Electronics Engineering, Shri Ramdeobaba College of Engineering and Management, Nagpur Email: ranepp1@rknec.edu,dawaresg@rknec.edu
}

Received: 20th November 2019, Accepted: 31st January 2020, Published: 30th April 2020

\begin{abstract}
Paper addresses Channel Estimation by means of Compressive Sensing (CS). Here system under consideration is OFDM. Paper reviews the emerging techniques of pilot design and allocation schemes. With the said pilot allocation techniques performance improvement in Mean square Error (MSE) and Bit Error Rate (BER) is achieved. Spectral efficiency can be considerably improved with channel estimation exploiting compressive sensing. Prominent algorithms are designed to attain better estimation accuracy even with reduction in pilot subcarrier figure. Comparatively simple CS structures with least computation complexity also reviewed in the projected paper.
\end{abstract}

Keywords

OFDM, MSE, BER, CS, CE, MIMO.

\section{Introduction}

OFDM is a digital modulation technique; find application in many wireless and telecommunication standards. Being multicarrier modulation scheme, it is being used for fourth-generation (4G) mobile broadband standards and wireless-local- area-network standards .It offers more flexibility and robustness over frequency-selective channels. In the proposed paper channel estimation in OFDM system with CS is discussed. Now days compressive sensing is gaining the tremendous attraction of researchers. Remarkable research has been done in this regard. When signal is sparse, with compressive sensing few compressive measurements of original signal are suffice for exact or approximate recovery. Paper discusses pilot design and allocation schemes and CS structures with least computation complexity for efficient channel estimation performance.

\section{Methodology}

Effective pilot design scheme is possible with compressive sensing for channel estimation in sparse channel scenario in OFDM system to ease the transmitted overhead. Here selection of pilot tones is of prior importance as it considerably affects channel estimation performance. Cross Entropy Optimization method may be employed for optimizing the OFDM pilot pattern in sparse channel estimation situation with compressive sensing framework [1]. Cross-entropy optimization results in reduced computational complexity and improved accuracy in channel estimation procedure. One can obtained improved Mean Square Error (MSE) with CEO method compared with the equi-spaced pilot tones as well as random pilot tones.

Down link large scale MIMO system having conventional orthogonal pilot experiences excessive overhead. To overcome this, structured CS based pilot design scheme can be employed. In this design scheme, the same subcarrier is occupied by the pilots of different antenna. Structured subspace pursuit algorithm [4] is designed based on the above theory. Algorithm recovers multiple channels and offers low pilot overhead.

Optimal pilot pattern is obtained iteratively by constructing and sampling probability distribution model of best pilot indices by means of Estimation of distribution algorithm (EDA)[7]. Algorithm maintains current promising indices as well as sampled the new once; hence taking care of local minima.

CS sensing algorithm work well when pilot subcarrier locations are pseudo random .For equi-spaced pilot structures in system like LTE, An iterative CS algorithm; A Practical Compressed Sensing Approach may be adopted [9]. Based on both the subcarrier level MSE of the CS algorithm and reliability of the soft/hard decisions; additional pilot utilization is decided.

With adaptive step size (AS-SaMP) algorithm one can adopt the adaptive step size adjustment approach to draw the exact sparsity level to ease the sparse channel estimation without knowing the channel sparsity level in advance. Further to guarantee a satisfactory estimation near-optimal pilot placement scheme is developed [10]. The optimal set of pilot locations is a cyclic difference set (CDS) on unitary discrete Fourier transform (DFT) matrix when minimizing the mutual coherence of the measurement matrix. Scheme may be restructured to $\mathrm{C}$ CDS with TS (concatenated cyclic difference set with an iterative tail search) in nonexistence scenario of CDS. To reduce coherence associated with pilot Fourier sub matrix, pilot locations as well as powers may be optimized with deterministic pilot design scheme for sparse channel estimation [13]. Scheme offers lower computational complexity. 
Deterministic pilot allocation of MIMO-OFDM systems is designed to better the performance of DCS-based channel estimation. For the said purpose genetic algorithm (GA)-based method of optimizing the pilot locations is offered; which results into the improved MSE for distributed compressed sensing DCS-SOMP-based channel estimate and the BER of the MIMO-OFDM or MISO-OFDM system [8]. Comparing CS-based and conventional with LS channel estimation methods and DCS with optimized pilot locations is proved to be better in both estimation performance and spectrum efficiency. Computational complexity on real-time realization does not result due to optimized pilot locations.

\section{Channel Estimation with Structured Compressive Scheme:}

For Multi Input Single output (MISO) OFDM downlink transmissions, CS employing a structured compressive scheme which utilizes impulse responses (CIRs) of channels [2].Utilizing block sparsity corresponding to aggregate CIR vector results in significant improvement in the estimation performance. Further to extract the channel parameters OMP (block-based Orthogonal Matching Pursuit) algorithm is developed. Scheme is effective in parameter estimation for all compassing antenna systems.

OFDM system with significant pilot figure estimates several channel parameters, results in reduced spectral efficiency. To conquer the issue Structured distributed CS estimation scheme for multiple symbol channels is developed [11].

Coefficient vectors of complex exponential basis expansion model (CE-BEM) are estimated; for this sparsity which is in delay domain contained by several OFDM symbols is exploited. ICI-free structure is obtained by designing pilot pattern based on sparsity within several OFDM symbols which results in a joint-block-sparse model. Coefficient vectors are precisely recovered together by orthogonal matching pursuit algorithm. By exploiting piecewise linear approximation, modeling error in CE-BEM is reduced. In terms of estimation accuracy SDCS-based scheme proved to be superior over conventional DCS-based and CS-based scheme when tracking the joint multi-symbol estimation model.

Channel estimation exploiting structured compressive sensing is designed for OFDM system for doubly selective channels. Where PN sequence which are in time-domain gives partial common support acquisition and the frequency-domain pilots realize the precise channel estimation [15]; ASA-BOMP algorithm is designed for this purpose.

\section{Channel Estimation exploiting Compressive Sensing with Low-Complexity:}

Priori-Information Aided Iterative Hard Threshold algorithm which is a high accuracy and low complexity scheme developed for TDS-OFDM Method compensate for the losses due to fading channels [5].First overlapadd technique is adopted for sequence training (time domain).Common estimation of channel parameters like path gain, delay and path length is performed by making use of channel's temporal correlation in fading and delay spread scenario resulting in strengthening the Channel estimation.

Integrated services digital broadcasting-terrestrial (ISDB-T) systems make use of CS for channel estimation [12]. Virtual oversampling is performed at the receiver to regulate successful reconstruction of the unknown CIR under the impact of fractional delay. Furthermore Multiple Orthogonal Matching Pursuit (MOMP) scheme is intended to diminish the computational complication caused by the virtual oversampling. Scheme is a modification of OMP. Reduction in bandwidth is achieved at transmitter side as oversampling is completed at receiver side. Bit error rate performance and computational complexity found to be better compared to a system without virtual oversampling

For reconstructing sparse signal CS is the most modern technique used. But it suffers with computational complications as it involves large size matrices for calculation. CS hardware with minimal complexity is realized for channel estimation in ISDB-T system. ISDB-T's basis is OFDM system [14]. For CS computation truncated DFT matrix forms the basis of computation matrix. Symmetrical property of DFT matrix helps in reduction of complications in multiplication and arbitrary memory access.

For rapidly time-varying channels in OFDM systems; the compressive channel estimator is developed; where on the basis of the non-diagonal matrix system model estimation is performed for doubly dispersive channel as a sparse recovery problem. Utilization of Modified-CS is done in each iterative step to lessen computational complications. based on a reasonable approximation of the measurement matrix [3].Method adopt reasonable approximation of the measurement matrix [3]. Further a specific pilot arrangement is added to obtain precision in the approximate system model.

\section{Channel Estimation with the Statistical Sparsity Profile of Signal:}

With Bayesian CS algorithm, reconstruction of UWB signal is achieved from a least number of collected random projection measurements. For CE eigen dictionary is developed by utilizing the statistical sparsity profile of UWB signals in the basis formed by eigen vectors. Expansion vector with respect to eigen dictionary is sparse and shows additional structure with statistically significant coefficients happening in clusters [6]. Algorithm can be advanced to recover multiple signals.

Multitask Bayesian compressive sensing (MBCS) is utilized to approximate the sparse channels of FDD massive MIMO-OFDM system. In real scenario it is assumed that sparsity level is unknown to base station. MBCS can jointly approximate the sparse angular channels of different subcarriers by making use of the spatial 
sparsity of massive MIMO channels considering the system bandwidth [16]. Further utilizing the sparse feature pilot adapted multitask bayesian compressive sensing MBCS (PAMBCS) scheme is designed, which proposes pilot sequences achieved with minimizing the differential entropy of approximated channel vectors, which results in reduction of estimation uncertainties. Even with few subcarrier number used in pilot transmission results in reduced pilot overhead with MBCS.

An adaptive structured-generalized orthogonal matching pursuit (AS-gOMP) algorithm for MIMO-OFDM Systems is a CS based channel estimation method [17]. Where initial sparsity and support set are obtained through rough estimation and the remaining support sets are acquired by the structured gOMP algorithm. Rough estimation and selecting $\mathrm{T}$ indices in each of it.

Result

Table No: 1 depicts Channel Estimation with Structured Compressive Scheme with algorithm used, system under consideration and observations.

\begin{tabular}{|c|c|c|c|c|}
\hline CE Scheme & $\begin{array}{c}\text { System } \\
\text { under } \\
\text { consideration }\end{array}$ & Algorithm & $\begin{array}{c}\text { Parameter } \\
\text { Evaluated for } \\
\text { Channel } \\
\text { Estimation }\end{array}$ & Observations \\
\hline $\begin{array}{l}\text { Structured } \\
\text { compressive CE } \\
{[2]}\end{array}$ & MISO-OFDM & $\begin{array}{l}\text { Block-based } \\
\text { OMP }\end{array}$ & $\begin{array}{l}\text { Normalized } \\
\text { mean square } \\
\text { error }\end{array}$ & $\begin{array}{l}\text { Achieves } 4 \mathrm{~dB} \text { gain compared to } \\
\text { conventional method. For a system } \\
\text { with large number of antenna with } \\
\text { shorter length preamble signal, } \\
\text { accurate CE is gained. }\end{array}$ \\
\hline $\begin{array}{l}\text { Structured } \\
\text { distributed } \\
\text { CS (SDCS) } \\
\text { based CE. CE } \\
\text { BEM model } \\
\text { exploited [11] }\end{array}$ & OFDM & $\begin{array}{l}\text { Block-based } \\
\text { SOMP }\end{array}$ & NMSE, BER & $\begin{array}{l}\text { Achieves accurate CE compared } \\
\text { with DCS and CS based schemes. }\end{array}$ \\
\hline $\begin{array}{l}\text { Doubly selective } \\
\text { CE based on } \\
\text { SCS [15] }\end{array}$ & TFT-OFDM & ASA-BOMP & MSE & $\begin{array}{l}\text { Probability of getting correct } \\
\text { recovery in terms of MSE less } \\
\text { than } 10^{-2} \text { is compared with BOMP } \\
\text { system. Proposed system } \\
\text { achieves } 2.5 \mathrm{~dB} \text { and } 5 \mathrm{~dB} \text { gain. }\end{array}$ \\
\hline
\end{tabular}

Table No 1: Channel Estimation with Structured Compressive Scheme

Table No: 2 depicts Channel Estimation exploiting Compressive Sensing with Low-Complexity.

\begin{tabular}{|l|l|l|l|l|}
\hline \multicolumn{1}{|c|}{ CE Scheme } & $\begin{array}{l}\text { System under } \\
\text { consideration }\end{array}$ & Algorithm & $\begin{array}{c}\text { Parameter } \\
\text { Evaluated for } \\
\text { Channel } \\
\text { Estimation }\end{array}$ & \multicolumn{1}{|c|}{ Observations } \\
\hline $\begin{array}{l}\text { Low complexity, } \\
\text { based CE[5] }\end{array}$ & TDS-OFDM & PA-IHT & $\begin{array}{l}\text { Spectral } \\
\text { efficiency }\end{array}$ & $\begin{array}{l}\text { Achieves higher spectral } \\
\text { efficiency than DPN-OFDM } \\
\text { scheme.Offers more accurate CE } \\
\text { than CoSaMP algorithm with } \\
\text { minimum computational } \\
\text { complexity. }\end{array}$ \\
\hline $\begin{array}{l}\text { low-complexity } \\
\text { CS } \\
\text { dimemain CE)[12] }\end{array}$ & $\begin{array}{l}\text { OFDM-based } \\
\text { DTTB }\end{array}$ & MOMP & NMSE,BER & $\begin{array}{l}\text { Reduction in computational } \\
\text { complexity and cost observed } \\
\text { with MOMP algorithm. Virtual } \\
\text { oversampling improves system } \\
\text { performance without increasing } \\
\text { bandwidth. }\end{array}$ \\
\hline
\end{tabular}




\begin{tabular}{|l|l|l|l|l|}
\hline $\begin{array}{l}\text { low-complexity } \\
\text { CS: Hardware } \\
\text { realization[14] }\end{array}$ & ISDB-T OFDM & OMP & BER & $\begin{array}{l}\text { Reduction in computational } \\
\text { complexity achieved with unique } \\
\text { matrix structure . Realized } \\
\text { Hardware achieves same BER } \\
\text { that with conventional method. }\end{array}$ \\
\hline CS-based CE[3] & OFDM & MOMP & MSE,BER & $\begin{array}{l}\text { Utilization of modified-CS results } \\
\text { in reduced computational } \\
\text { complexity. Perform well with } \\
\text { fast time varying channels } \\
\text { compared to conventional one. }\end{array}$ \\
\hline
\end{tabular}

Table No 2: Channel Estimation exploiting Compressive Sensing with Low-Complexity

Table No: 3 depicts Channel Estimation with the Statistical Sparsity Profile of Signal

\begin{tabular}{|c|c|c|c|c|}
\hline CE Scheme & $\begin{array}{c}\text { System } \\
\text { under } \\
\text { considerati } \\
\text { on }\end{array}$ & Algorithm & $\begin{array}{l}\text { Parameter } \\
\text { Evaluated for } \\
\text { Channel } \\
\text { Estimation }\end{array}$ & Observations \\
\hline $\begin{array}{l}\text { CS for USB } \\
\text { Channel } \\
\text { Estimation[6] }\end{array}$ & $\begin{array}{l}\text { UWB } \\
\text { signals }\end{array}$ & $\begin{array}{l}\text { Bayesian } \\
\mathrm{CS}, \quad \mathrm{MT}- \\
\mathrm{BCS}\end{array}$ & BER & $\begin{array}{l}\text { Algorithm reduces required } \\
\text { sampling rate and offers improved } \\
\text { performance over conventional CS- } \\
\text { based schemes. }\end{array}$ \\
\hline $\begin{array}{l}\text { Pilot Adapted } \\
\text { Multitask } \\
\text { Bayesian CS } \\
\text { (PAMBCS)[16] }\end{array}$ & $\begin{array}{l}\text { MIMO- } \\
\text { OFDM }\end{array}$ & MBCS & $\begin{array}{l}\text { Differential } \\
\text { entropy, Pilot } \\
\text { overhead }\end{array}$ & $\begin{array}{l}\text { Even with few subcarrier number } \\
\text { used in pilot transmission results in } \\
\text { reduced pilot overhead with MBCS. }\end{array}$ \\
\hline $\begin{array}{l}\text { Time domain } \mathrm{CE} \\
{[17]}\end{array}$ & $\begin{array}{l}\text { MIMO- } \\
\text { OFDM }\end{array}$ & AS-gOMP & BER & $\begin{array}{l}\text { Improved performance and reduced } \\
\text { BER is achieved with proposed } \\
\text { method [17] exploiting the PN } \\
\text { sequence characteristics. }\end{array}$ \\
\hline
\end{tabular}

Table No 3: Channel Estimation with the Statistical Sparsity Profile of Signal

\section{Conclusion and Future Scope}

For sparse Channel Estimation in OFDM system, an competent pilot design scheme is possible with application of compressive sensing to ease the transmitted overhead. One can obtained improved MSE of channel estimation using CEO method compared with the equi-spaced pilot tones and random pilot tones [1].SSP algorithm [4] based on theory of structured compressive sensing (CS) recovers multiple channels with reduced pilot overhead. Algorithm can be completed for uplink large scale MIMO system and is also applicable for conservative small-scale MIMO systems for defined CSI acquisition with reduced pilot overhead. Comparing CS-based and conventional with LS channel estimation technique and distributed compressed sensing (DCS) with optimized pilot positions is proved to be better in both estimation performance and spectrum efficiency [8]. Bayesian CS algorithm [6] can be advanced to recover multiple signals.

\section{References}

[1] An Efficient Pilot Design Scheme for Sparse Channel Estimation in OFDM Systems; Jung-Chieh Chen, Member, IEEE, Chao-Kai Wen, Member, IEEE, and Pangan Ting, Member, IEEE, IEEE communications letters July 2013.

[2] Structured Compressive Channel Estimation for Large-Scale MISO-OFDM Systems Weikun Hou and Chia Wei Lim, IEEE communications letters, VOL. 8, NO. 5, May 2014765.

[3] Two compressive sensing-based estimation schemes designed for rapidly time-varying channels in orthogonal frequency division multiplexing systems;Yi Liu,2, Wenbo Mei, Huiqian DuPublished in IET Signal Processing, doi: 10.1049/iet-spr.2013.0352,Special Issue on Compressive Sensing and Robust Transforms [4 ] Structured compressive sensing based superimposed pilot design in downlink large-scale MIMO systems;Zhen Gao, Linglong Dai and Zhaocheng Wang,ELECTRONICS LETTERS 5th June 2014 Vol. 50 No. 12 pp. 896-898. 
[5]Priori-Information Aided Iterative Hard Threshold: A Low-Complexity High-Accuracy Compressive Sensing Based Channel Estimation for TDS-OFDM;Zhen Gao, Chao Zhang, Member, IEEE, Zhaocheng Wang, Senior Member, IEEE, and Sheng Chen, Fellow, IEEE IEEE transactions on wireless communications, vol. 14, no. 1, January 2015.

[6] Ultra wideband Channel Estimation: A Bayesian Compressive Sensing Strategy Based on Statistical Sparsity;Xiantao Cheng, Member, IEEE, Mengyao Wang, and Yong Liang Guan, Member, IEEE, IEEE transactions on vehicular technology, vol. 64, no. 5, may 20151819.

[7] Pilot Pattern Optimization for Sparse Channel Estimation in OFDM Systems;Han Wang, Qing Guo, Member, IEEE, Gengxin Zhang, Guangxia Li, and Wei Xiang, Senior Member, IEEE, IEEE communications letters, vol. 19, no. 7, July 20151233.

[8] Pilot Allocation for Distributed-Compressed-Sensing-Based Sparse Channel Estimation in MIMO-OFDM Systems; Xueyun He, Rongfang Song, and Wei-Ping Zhu, Senior Member, IEEE, IEEE transactions on vehicular technology, vol. 65, no. 5, May 2016.

[9] A Practical Compressed Sensing Approach for Channel Estimation in OFDM Systems;Istdeo Singh, Sheetal Kalyani, and K. Giridhar, IEEE communications letters, vol. 19, no. 12, December 2015.

[10] Novel Compressed Sensing-Based Channel Estimation Algorithm and Near-Optimal Pilot Placement Scheme;Yi Zhang, Ramachandran Venkatesan, Senior Member, IEEE, Octavia A. Dobre, Senior Member, IEEE, and Cheng Li, Senior Member, IEEE; IEEE transactions on wireless communications, vol. 15, no. 4, April 2016.

[11] Structured Distributed Compressive Channel Estimation Over Doubly Selective Channels; Qibo Qin, Lin Gui, Member, IEEE, Bo Gong, Xiang Ren, and Wen Chen, Senior Member, IEEE; IEEE transactions on broadcasting, vol. 62, no. 3, September 2016521.

[12] Low-Complexity Compressed Sensing-Based Channel Estimation With Virtual Oversampling for Digital Terrestrial Television Broadcasting;Ryan Paderna, Duong Quang Thang, Member, IEEE, Yafei Hou, Senior Member, IEEE,Takeshi Higashino, Member, IEEE, and Minoru Okada, Member, IEEE; IEEE transactions on broadcasting.

[13]Compressive Sensing-Based Pilot Design for Sparse Channel Estimation in OFDM Systems; Roozbeh Mohammadian, Arash Amini, Senior Member, IEEE, and Babak Hossein Khalaj, Member, IEEE; IEEE communications letters, vol. 21, no.1, January 2017.

[14] A Low-Complexity Hardware Implementation of Compressed Sensing-Based Channel Estimation for ISDB-T System:Rian Ferdian, Student Member, IEEE, Yafei Hou, Senior Member, IEEE, and Minoru Okada, Member, IEEE; ieee transactions on broadcasting 1 .

[15] Structured Compressive Sensing Based Channel Estimation for Time Frequency Training OFDM Systems over Doubly Selective Channel:Xu Ma, Fang Yang, Senior Member, IEEE, Sicong Liu, Wenbo Ding, and Jian Song, Fellow, IEEE; DOI 10.1109/LWC.2017.2669974, IEEE Wireless Communications Letters.

[16]Common Sparsity based Channel Estimation for FDD Massive MIMO-OFDM Systems via Multitask Bayesian Compressive Sensing ;Wei Ji| Ling Qiu,2017 IEEE 86TH Vehicular Technology Conference(VTCFall),2017.

[17] Compressive Sensing Based Channel Estimation for MIMO-OFDM Systems ;Weisi Kong,Hui Li,Shuangshuang Song,Yujie Fan,Wenjie Zhang,13th IEEE conf. on Industrial Electronics and application(ICIEA)2018. 